\title{
POLÍTICAS E MODELOS ASSISTENCIAIS EM SAÚDE E REABILITAÇÃO DE PESSOAS PORTADORAS DE DEFICIÊNCIA NO BRASIL: ANÁLISE DE PROPOSIÇÕES DESENVOLVIDAS NAS ÚLTIMAS DUAS DÉCADAS*
}

\author{
POLICIES AND ASSISTENCIAL MODELS IN \\ DISABLED'S HEALTH AND REHABILITATION IN \\ BRAZIL: ANALISYS ABOUT TWO DECADES
}

\author{
Marta Carvalho de Almeida ${ }^{(1)}$, \\ Gastão Wagner Souza Campos ${ }^{(2)}$
}

\begin{abstract}
ALMEIDA, M. C. de.; CAMPOS, G. W. S. Políticas e modelos assistenciais em saúde e reabilitação de pessoas com deficiência no Brasil: análise de proposições desenvolvidas nas últimas duas décadas. Rev. Ter. Ocup. Univ. São Paulo, v. 13, n. 3, p. 118-26, set./dez. 2002.

RESUMO: Embora nas últimas décadas as pessoas portadoras de deficiência tenham realizado conquistas importantes no tocante à legislação, as políticas públicas brasileiras não ofereceram suporte e possibilidades de consolidação do exercício de seus direitos sociais, entre eles o direito à saúde e reabilitação. O estudo desenvolvido enfocou políticas e modelos assistenciais em saúde e reabilitação de pessoas portadoras de deficiência física instituídos no cenário assistencial brasileiro nos últimos 20 anos, com o objetivo de reconstruir e analisar seus percursos práticos e conceituais. Com base em dados coletados na literatura e em entrevistas, enfocou o modelo hegemônico, ou seja, aquele que concentra as ações no Centro de Reabilitação, e a Reabilitação Baseada na Comunidade - RBC, constituída como modelo alternativo às práticas institucionais, pela Organização Mundial de Saúde - OMS. No artigo houve opção por apresentar resultados que se articulam às questões que envolvem o desenvolvimento da atenção à saúde e reabilitação no Sistema Único de Saúde - SUS. Assim, a apresentação da análise dos dois modelos - que resultou na consideração de que não se produziu, no período, políticas e modelos assistenciais que tenham alterado significativamente a transferência de recursos públicos para o setor privado, bem como superado os limites do paradigma biomédico - leva à discussão acerca de proposições detentoras de potencial para gerar transformações na atenção à saúde e reabilitação de pessoas portadoras de deficiência no âmbito público. Aponta-se a necessidade e viabilidade de ampliar o objeto de ação da reabilitação, invocando noções da Reabilitação Psicossocial que instigam à flexibilidade e diversificação dos serviços, à realização de ações voltadas à prevenção ou desmonte de processos de exclusão social e à ativação de recursos comunitários não aparentes ou formalmente instituídos.
\end{abstract}

DESCRITORES: Pessoas deficientes/reabilitação. Formulação de políticas. Reabilitação/ tendências. SUS (BR). Apoio social. Prestação de cuidados de saúde.

O artigo apresenta parte da pesquisa desenvolvida para obtenção do título de doutor em Saúde Coletiva, cujo título é "Saúde e reabilitação de pessoas com deficiência: políticas e modelos assistenciais", defendida na Faculdade de Ciências Médicas da UNICAMP em 2000..

(1) Profa. Dra. do Curso de Terapia Ocupacional do Departamento de Fisioterapia, Fonoaudiologia e Terapia Ocupacional da Faculdade de Medicina da Universidade de São Paulo. Doutora em Saúde Coletiva pela Faculdade de Ciências Médicas da UNICAMP.

(2) Doutor em Saúde Coletiva, Prof. Associado do Departamento de Medicina Preventiva e Social da Faculdade de Ciências Médicas da UNICAMP.

Endereço para correspondência: Departamento de Fisioterapia, Fonoaudiologia e Terapia Ocupacional da Faculdade de Medicina da Universidade de São Paulo. Rua Cipotânea, 51 Cidade Universitária "Armando Salles de Oliveira". 05360-000. São Paulo, SP. e-mail: mcarmei@usp.br 
$\mathrm{N}$ o Brasil, fatos ocorridos nas décadas de 1980 e 1990 são referências que marcam o processo de consolidação dos direitos de pessoas portadoras de deficiência. No contexto de redemocratização do país, na década de 1980, a ação organizada dessa parcela da população gerou, ao lado de outros atores sociais, importantes conquistas na esfera institucional, essencialmente no campo da legislação. Pessoas portadoras de deficiência foram discriminadas positivamente em vários pontos da Constituição promulgada em 1988, e a Lei n. 7853/89 responsabilizou o poder público a assegurar o pleno exercício de seus direitos, tais como os direitos à educação, à saúde, ao trabalho e ao lazer, entre outros.

Essas conquistas, porém, não barraram o avanço dos processos sociais de exclusão que, em sentido oposto, aprofundaram as contradições na sociabilidade desse segmento. Se, por um lado, a legislação garantiu a participação na produção e partilha de bens, serviços e valores sociais, por outro, tal como ocorreu com outros segmentos sociais, verificou-se a ausência de políticas que oferecessem suporte ao real exercício desses direitos.

No campo da saúde, especificamente, nessas décadas projetaram-se mudanças importantes para pessoas portadoras de deficiência, na medida em que os princípios de construção do Sistema Único de Saúde - SUS apontaram para uma efetiva inclusão desse grupo no conjunto de usuários do sistema. A universalidade e a hierarquização, propostas como diretrizes, a valorização de ações de prevenção e a mudança do modelo hospitalocêntrico, bem como outras proposições, alimentaram a esperança de que, finalmente, as demandas desse grupo motivariam a elaboração e concretização de ações de saúde e reabilitação na esfera pública, ampliando e qualificando significativamente a oferta de atenção. Delineou-se o caminho da superação da baixíssima cobertura assistencial oferecida, bem como da vigência do assistencialismo, instituído como "atenção" através das poucas mas predominantes instituições filantrópicas que atuavam na área (BRASIL, 1995).

Porém, passados mais de 10 anos do reconhecimento dos deveres do poder público quanto à saúde e reabilitação, não foram evidentes alterações significativas no cenário assistencial brasileiro, bem como nas precárias condições de vida e saúde da maior parte das pessoas portadoras de deficiência, conforme apontou a Organización Panamericana de la Salud OPS (1996).

O estudo realizado, investigando esse período, tomou políticas e modelos assistenciais como objeto de análise. Embora compreendendo que a inclusão social de pessoas portadoras de deficiências como objeto de intervenções não esteja limitado ao campo específico da saúde, devendo envolver diferentes setores implicados na elaboração de respostas a essa problemática, considerou-se a saúde um campo especialmente relevante. Afinal, a medicina assumiuse, em determinado período da história, como universo preferencial de desenvolvimento de práticas cujo objetivo declarado foi o de produzir a reintegração de pessoas com deficiência na sociedade (DE LA BELLACASA, 1990).

Assim, buscou-se compreender como vem sendo pensada e proposta a produção de serviços de saúde e reabilitação de pessoas portadoras de deficiência no Brasil nos últimos 20 anos - mais especificamente daqueles que se voltam às incapacidades motoras. Adotou-se referenciais da Saúde Coletiva a fim de analisar os percursos conceituais, as diretrizes operacionais e algumas das ações práticas realizadas e documentadas nesse período.

\section{PERCURSO METODOLÓGICO}

Foram definidos para estudo o modelo representado pelo Centro de Reabilitação-CR, ou seja, o modelo hegemônico de reabilitação no Brasil, e a Reabilitação Baseada na Comunidade - RBC, que foi criada e recomendada pela Organização Mundial de Saúde - OMS aos países em processo de desenvolvimento econômico, a partir da década de 1980. Os critérios usados para a definição desses modelos consideraram necessária a apresentação de um conjunto organizado de pressupostos, conceitos e diretrizes políticas e operacionais, além da existência real no cenário nacional de atenção às pessoas portadoras de deficiência.

Verificou-se, também, a pertinência de considerar, no estudo, o programa proposto pelo Ministério da Saúde em 1994, notando que, embora não tenha sido implementado de forma abrangente, o programa documenta o reconhecimento da precariedade da atenção a esse segmento da população e propõe medidas para saná-la no contexto de construção do SUS.

A coleta de dados aconteceu sobre diferentes fontes escritas: prioritariamente artigos publicados em periódicos científicos, livros, relatórios e pareceres de órgãos oficiais, e também alguns materiais de divulgação e textos disponibilizados em rede.

Convém esclarecer que devido ao fato de reunirem-se sob a denominação "Reabilitação Baseada na Comunidade" práticas diversas, optou-se por 
delimitar o campo de experiências para estudo. Para isso, tomou-se a raiz histórica da RBC, considerando que $a$ adoção de diretriz política relativa à ampliação de cobertura, associada à redução de custos e desenvolvimento de ações em área geográfica delimitada, através de implementação de procedimentos simplificados, realizados por pessoal não-profissional capacitado pelo próprio programa, corresponde à definição de práticas que podem, inequivocamente, serem denominadas de RBC.

Foram, também, colhidos depoimentos de pessoas que protagonizaram a história recente da reabilitação no país, particularmente no Estado de São Paulo, tendo ocupado funções de gerência ou planejamento das políticas de atenção no período estudado, através de entrevista do tipo semi-estruturado.

Adotou-se, para produzir a análise, a categoria modelos assistenciais, com definição elaborada por Campos (1992), que propõe que a forma de produção de serviços - termo empregado como sinônimo de modelo assistencial - seja entendida como o resultado de uma composição concreta de recursos, tecnologias e modalidades de atenção, que se articulam em um dado contexto histórico-estrutural em busca de legitimidade e de reprodução social. Os recursos são financeiros, materiais e a força de trabalho, e as modalidades de atenção são as várias partes que, combinadas segundo o sentido determinado pela totalidade do modelo, constituem-no. Essa composição gera, portanto, uma dada estrutura produtiva e um certo discurso que são passíveis de análise.

\section{RESULTADOS}

Divulgar resultados de estudos qualitativos de forma condensada é sempre uma tarefa difícil. Produzir sínteses, ou recortes essenciais, implica em selecionar apenas um, entre tantos enfoques possíveis. Assim, a opção deste artigo foi ressaltar, do estudo realizado, os resultados que podem ser relacionados de maneira mais prática e direta à discussão sobre a reabilitação no SUS.

\section{O MODELO HEGEMÔNICO DE REABILITAÇÃO}

A análise do curso histórico da reabilitação no Brasil mostra que a atenção a pessoas portadoras de deficiências físicas teve impulso significativo no final da década de 1950, quando passou a ser consistentemente orientada, prática e ideologicamente, pelas concepções médicas sobre as incapacidades e a reabilitação física. Mediante a implantação do Centro de Demonstração de Técnicas de Reabilitação da OMS - o Instituto Nacional de Reabilitação, INAR - instalado no Hospital das Clínicas da Faculdade de Medicina da Universidade de São Paulo, em 1956, deu-se início à visibilidade e legitimação social de um modelo de atenção em reabilitação concentrado na instituição Centro de Reabilitação. Sua configuração seguiu o modelo de reabilitação vigente nos países desenvolvidos economicamente, no qual caberia à instituição ser o lugar específico da reabilitação. As pessoas portadoras de deficiência integraram essas novas concepções não como sujeitos de direito, mas como indivíduos dotados de potencial funcional para a reintegração à sociedade. O desenvolvimento desse potencial seria, então, a conseqüência lógica da aplicação das técnicas de intervenção da reabilitação física.

Para compor essa instituição, portanto, previramse recursos materiais especiais, principalmente destinados à avaliação e intervenção sobre funções motoras, e equipe técnica agregando especialistas de outras profissões, coordenados pelo médico. Essa é, até os dias atuais, sua configuração básica.

As produções críticas acerca desse modelo, ou seja, da reabilitação praticada em instituições médicoespecializadas, mostram que a racionalidade médica, condicionando a organização do trabalho da equipe e as modalidades de atenção à pessoa, instituiu a melhora ou aumento do desempenho funcional como missão quase exclusiva, abordando redutivamente os processos que envolvem a vida com deficiência conforme apontam Rocha (1990); Almeida (1993); Nallin (1992), entre outros. Esses autores mostram ter havido uma pressuposição de que a normalização de funções corporais seria a condição requerida para a integração social, excluindo do foco de atenção da reabilitação inúmeros processos sociais e demandas significativas para a pessoa. Articuladamente, a propagação da idéia de que a integração social dependeria, fundamentalmente, de conquistas individuais, contribuiu para a legitimação social do modelo, ainda que este não realize, de fato, o que propõe. Nallin (1992), além desses aspectos, mostra que sob a declarada neutralidade científica dessas ações técnicas, orientadas a corrigir um corpo-objeto, subjaz uma dimensão política cujo resultado é a subtração do sujeito de sua capacidade de autodeterminação e, portanto, das possibilidades de participação social em condições de igualdade frente ao grupo social ao qual pertence. Nos estudos desenvolvidos no campo da reabilitação médica não há referências à inclusão ou formas de participação social, na medida em que predominam as investigações voltadas às capacidades funcionais dos sujeitos. 
Verifica-se na literatura que é essencialmente nesse âmbito que se situam o interesse e as realizações da reabilitação médico-especializada, progressivamente mais associados a tecnologias sofisticadas que permitem diagnósticos e resultados mais precisos e mais localizados.

Soares (1987), considerando o período compreendido pelas décadas de 1950 a 1980 , tratou da introdução e difusão dessa orientação ideológica na atenção à pessoa portadora de deficiência no Brasil, enfocando-as tanto da perspectiva previdenciária quanto da ideologia assistencialista. Apontou que uma das ações mais significativas do Estado na reabilitação ocorreu na forma de incentivos, inclusive financeiros, à criação de serviços privados com ou sem fins lucrativos, dos quais passou a comprar ações de reabilitação através de convênios. Parte considerável dessas unidades assistenciais desenvolveram o amálgama entre a caridade e a ciência descrito por Nallin (1992), mesclando concepções religiosas e técnicas no âmbito das estruturas física, organizacional e relacional das instituições. Nas unidades lucrativas adaptou-se o modelo médico - originalmente associado a estruturas hospitalares - dando origem a clínicas de médio e pequeno porte constituídas nos moldes dos CRs.

O estudo proposto verificou que no período analisado houve persistência dessa forma de ação estatal. Na medida que o Estado não tem promovido de maneira significativa, através de suas unidades próprias, as ações necessárias ao suprimento da oferta de atenção em reabilitação, continua prevalecendo a política que transfere recursos públicos para instituições privadas, conforme discutiu Oliver (1998).

Pode-se notar, ainda, que além das instituições de reabilitação serem numericamente insuficientes, a exclusão de extensos segmentos de demandantes foi causada, também, pelas rigorosas normas de ingresso e de adesão aos programas desenvolvidos. A definição dessas normas parece obedecer, mais freqüentemente, aos interesses próprios à instituições. Embora dependentes de recursos públicos, essas instituições resistiram a orientações, compromissos ou processos de avaliação definidos pela esfera pública.

Assim, mesmo após algumas décadas de debates, nos quais diferentes grupos e organismos que se ocupam de refletir e propor diretrizes políticas para a área, apontam a inadequação e insuficiência do modelo assistencial concentrado no CR, ele prevalece no Brasil. Ainda são poucas as unidades públicas que atendem às demandas de pessoas com deficiência - sendo freqüentemente ligadas a universidades - o que confirma que a universalidade do SUS não é, ainda, uma realidade para essas pessoas.
A hierarquização do sistema de saúde, no que tange à saúde e reabilitação de pessoas portadoras de deficiência, encontra também forte resistência à sua consolidação, na medida em que da perspectiva médica - que representa essencialmente o grupo mais forte entre os atores políticos da área - o CR continua sendo a instância onde devem se concentrar todos os recursos destinados a prevenir e reabilitar pacientes portadores de doenças potencialmente incapacitantes e de deficiências, sendo estas transitórias ou definitivas. Constatou-se que, recentemente, mesmo entre os mais resistentes, essa idéia vem dividindo espaço com considerações sobre a desconcentração da atenção, sugerindo certa adesão aos princípios do SUS e transformação do pensamento original. Observa-se, porém, que embora se admita a necessidade de outras unidades integrarem um sistema de atenção à saúde da pessoa com deficiência, mantém-se a concentração de poder no $\mathrm{CR}$, visto que este continuaria definindo com exclusividade o projeto terapêutico a ser desenvolvido junto ao paciente. Dessa perspectiva, profissionais situados em outras unidades de saúde seriam comandados por aqueles do CR e responsáveis, unicamente, por oferecer "seguimento" à pessoa previamente atendida no CR. São ainda previstas ações executadas por profissionais não-especializados, em algumas situações, para evitar que seja obrigatório deixar a comunidade para dar continuidade à assistência.

\section{A REABILITAÇÃO BASEADA NA COMUNIDADE: DESLOCAMENTOS TEÓRICO- PRÁTICOS NA REABILITAÇÃO}

Parte significativa das críticas ao modelo de reabilitação concentrado na instituição também foram produzidas pela OMS e a OPAS. Em meados da década de 1970, ao mesmo tempo em que recomendavam novas diretrizes a serem aplicadas nas décadas seguintes em relação às políticas globais de saúde, deram tratamento específico para a área de reabilitação, afinando-a às primeiras. Assim, o deslocamento do foco institucional para a comunidade se faria sentir também na reabilitação.

Essas organizações, nesse período, não apenas adotaram um tom crítico em relação aos Estados omissos em relação ao problema das incapacidades, como também prestaram-se a formatar modelos alternativos aos CRs - considerados inviáveis, do ponto de vista econômico, para os países economicamente instáveis, onde ocorreria a maior incidência de casos. A ausência ou ineficácia das instituições de reabilitação 
nos países em processo de desenvolvimento econômico, bem como a super-concentração de recursos em instituições especializadas, foram argumentos extensamente apresentados. (HELANDER et al., 1980; OMS, 1981; OPAS, 1989, 1993; ONU, 1992). A aproximação da reabilitação com a comunidade, seja através de sua inserção na Atenção Primária, seja através da implementação de ações consubstanciadas no projeto denominado Reabilitação baseada na Comunidade $R B C$, foi defendida como princípio de adequação às reais necessidades de pessoas portadoras de deficiência. Assim, o objetivo principal da RBC seria o de responder a essas necessidades através da utilização de recursos humanos e materiais presentes na própria comunidade, valorizando a criação de soluções locais, substitutivas ao uso de tecnologias sofisticadas e onerosas consideradas desnecessárias para responder à maior parte das demandas. Preconizou-se a transferência de poder e responsabilidade às comunidades pela atenção e inclusão social de seus membros portadores de deficiência, adotando-se a perspectiva de que os projetos de RBC tornar-se-iam auto-sustentáveis, após um período inicial de apoio.

Nesse contexto de redefinições de atribuições e papéis, o CR passou a ser divulgado, por essas organizações, como instituição de importância irrefutável. Porém, como estrutura destinada a desenvolver ações que se prestam a resolver aspectos pontuais do processo de reabilitação, e não todo ele (PEAT, 1997).

No Brasil, essas mudanças de enfoque, articuladas aos movimentos sociais, produziram um conjunto de proposições políticas importantes, cuja concretização ocorreu mais freqüentemente em âmbito municipal ou comunitário. Desse conjunto, destaca-se por relevância e abrangência, o Programa de Atenção à Saúde da Pessoa Portadora de Deficiência do Ministério da Saúde, de 1994, que adotou como objetivo a atenção integral da pessoa portadora de deficiência no SUS, delimitando níveis de complexidade e descrevendo ações de prevenção, detecção precoce, diagnóstico, tratamento, reabilitação e integração social. No programa, a RBC é tratada como modelo assistencial orientado por concepções distintas da reabilitação institucional e é indicada como estratégia a ser desenvolvida no contexto da Atenção Primária.

A partir das recomendações da OMS na década de 1980, várias experiências foram desenvolvidas em diferentes regiões do mundo*. Essas experiências receberam estímulo para desenvolver procedimentos de avaliação de resultados, bem como para manifestaremse acerca do modelo proposto, já que este foi apresentado em manual ${ }^{* *}$, cuja elaboração coube a Helander et al. (1980).

Acompanhando essas experiências, vários aspectos da RBC vêm sendo alvo de investigações e alimentam reflexões importantes. A literatura consultada permite algumas constatações:

- os projetos apoiados pela OMS e OIT situamse, basicamente, nos países em processo de desenvolvimento econômico. Além de apoiarem técnica e/ou financeiramente parte significativa desses projetos, esses organismos também tem sido os principais responsáveis por sua divulgação ampliada. Em grande parte dos projetos há intercâmbio técnico e científico com importantes centros de estudos estrangeiros, implicados diretamente com o desenvolvimento das ações;

- o caráter público da RBC não se consolidou. Na maior parte das experiências, os Estados aceitaram contribuir com projetos de RBC que traziam recursos externos, mas não a implementaram como política nacional;

- os projetos de RBC são bastante diferentes entre si, seguindo vezes mais, vezes menos, a estrutura proposta pela OMS. A heterogeneidade do campo produziu diversos pontos de vista acerca de temas específicos. Principalmente quanto ao papel a ser atribuído à comunidade e às pessoas com deficiência

\footnotetext{
"O cap. 3 "Comunidade e participação: a crise do modelo hegemônico e a constituição de propostas alternativas", da tese "Saúde e Reabilitação de pessoas com deficiência: políticas e modelos assistênciais", reúne dados de parte dessas experiências internacionais e nacionais.

** O manual serve, em essência, como estratégia de implantação do modelo, através da capacitação da comunidade. Espera-se que, mediante treinamento, supervisores locais, líderes comunitários e professores, além das próprias pessoas com deficiência, familiares e cuidadores, desenvolvam ações de reabilitação na comunidade, de forma não-remunerada. Essas pessoas pertenceriam a dois, dos três níveis descritos no modelo: o nível intermediário e o nível comunitário. O supervisor local - pertencente ao nível intermediário - poderá ser um trabalhador de saúde de nível médio ou básico, que terá a tarefa de selecionar os participantes que atuarão com cada paciente sob sua responsabilidade, instruir e supervisionar aqueles que não são profissionais - sejam eles voluntários, familiares, cuidadores ou pessoas com deficiência - avaliar os resultados e relacionar-se com os profissionais de níveis superiores ao seu. Aos líderes comunitários o manual quer mostrar a possibilidade de convivência social com pessoas com deficiência fundamentada na igualdade, bem como as formas de integrá-los e promover seu acesso às mesmas oportunidades oferecidas aos outros cidadãos da comunidade. Os professores recebem orientação de como facilitar a inclusão de crianças com deficiência em escolas de ensino regular.
} 
no projeto, e quanto à missão das estruturas de nível local, intermediário e especializado na implementação do modelo;

- algumas experiências de RBC vem ocorrendo no Brasil, geralmente localizadas em zonas rurais ou em pontos da periferia de grandes cidades. Essas experiências, que geralmente se apresentam de forma pouco identificada ao modelo estrutural da OMS, não se inserem no grupo de projetos diretamente apoiados por essa organização e, mais freqüentemente, ocorrem mediante parcerias da comunidade com organizações não-governamentais nacionais e internacionais. Várias são marcadas pela ausência de recursos suficientes para atingir de forma adequada os problemas, dada a complexidade destes;

- a RBC tem estimulado o desenvolvimento de inquéritos domiciliares ou estudos censitários sobre as deficiências e os problemas enfrentados por essas pessoas na comunidade em que vivem. Os resultados freqüentemente revelam aspectos novos e significativos para a elaboração de projetos de atenção;

- as experiências de RBC promovidas pela OMS orientaram-se pelo paradigma biomédico, não tendo alterado, substantivamente, a racionalidade da reabilitação. Profissionais especializados, atuantes nos CRs, têm sido os principais transmissores de conhecimento à comunidade, que acaba por reproduzir determinados esquemas de entendimento e de ação em relação às deficiências, criando-os com menor frequiência. As experiências que mais produziram práticas terapêuticas inovadoras foram aquelas mais solidamente, e prioritariamente, articuladas aos coletivos bem organizados e representativos dos interesses das pessoas portadoras de deficiência;

- os resultados da RBC avaliados de maneira mais positiva dizem respeito a mudanças de atitude da comunidade em relação às pessoas portadoras de deficiência;

- aparecem, pontuados nos relatos de experiências, vários resultados não relevantes do ponto de vista quantitativo, haja vista sua singularidade. Contudo, estão associados a mudanças altamente significativas na vida de determinadas pessoas ou famílias;

- a participação da comunidade tem ocorrido mais ao nível do cuidado direto das pessoas com deficiência do que em níveis de planejamento e decisão, o que tem sido apontado como uma falha da RBC. Ao mesmo tempo, evidenciou-se a capacidade de pessoas da comunidade para identificar e ativar recursos informais no entorno social;

- a falta de remuneração dos trabalhadores coloca problemas importantes, tanto na esfera política quanto na prática. Do ponto de vista prático, o engajamento profissional baseado essencialmente em critérios pessoais tem tornado instáveis o compromisso de trabalho e o padrão quantitativo de atividades realizadas;

- a necessidade de que sejam discriminados os problemas para os quais a RBC - tal como é proposta pela OMS - é melhor indicada, bem como em quais contextos ela deve ser desenvolvida, tem sido apontada nos documentos mais recentes;

- não se confirmaram as projeções acerca da autosustentabilidade da RBC. Não há projeto que tenha sobrevivido apenas com recursos locais. Como afirmam Momm e Konig (1990) e Crishna (1999) a boa reabilitação requer recursos.

\section{CONSIDERAÇÕES FINAIS E NOTAS SOBRE A REABILITAÇÃO NO SUS}

Verificou-se que nas últimas décadas não houve alterações significativas na política e no modelo assistencial de atenção à saúde e reabilitação de pessoas portadoras de deficiência no Brasil. Seus mecanismos de reprodução mantiveram-se vigentes e politicamente estáveis, tanto quanto persistiram os problemas dessa parcela da população. Embora a RBC criada pela OMS tenha gerado experiências práticas que dão materialidade a novos princípios e diretrizes para a área de reabilitação, tomando sob sua responsabilidade demandas ampliadas que não são enfocadas pelas práticas convencionais, o modelo não se desenvolveu de forma a produzir - e poder representar - a superação dos limites impostos pelo modelo médico de compreensão e intervenção sobre as deficiências. Tampouco foi integrado às políticas públicas ou mostrou-se eficiente enquanto alternativa à falta de recursos investidos na rede pública.

Contudo, as reflexões acerca das alternativas à reabilitação instituída, bem como os processos concretamente implementados no sentido de produzir atenção mais significativa no Brasil, apresentam um conjunto de inovações que poderia ser convertido em base potencialmente capaz de alavancar processos voltados a consolidar direitos da pessoa portadora de deficiência. A RBC, mais avaliada e investigada enquanto modelo de atenção do que o Centro de Reabilitação, foi responsável pela fomentação de um campo crítico-reflexivo importante. Nele está situada parte considerável das produções teórico-práticas relevantes para a busca de maior impacto nos problemas vividos por pessoas com deficiência. Alguns pontos 
podem dele ser destacados para apoiar a produção de novas formas de produzir serviços de reabilitação no SUS - inclusive nos CRs já existentes*.

Ampliar o objeto de ação da reabilitação, ou ainda, oferecer maior acolhimento aos diversos problemas enfrentados pelas pessoas portadoras de deficiência através da atenção à saúde e reabilitação, é necessário e viável. Admitir que a efetividade da reabilitação é condicionada por outras variáveis, diferentes da qualidade do desempenho funcional, poderia levar a que fossem tomados como alvo a prevenção ou o desmonte de complexos processos de exclusão social.

Adotar essa referência poderia ser uma estratégia importante para qualificar os projetos terapêuticos desenvolvidos nas unidades de atenção, tornando a intervenção algo que, prioritariamente, criaria condições para que as pessoas com deficiência exercitassem mais escolhas. O enfrentamento das diversas faces da exclusão social, ao representar um desafio às formas convencionais de produção de serviços, poderia conduzir à sua flexibilização e diversificação, tornando-os lugares permeáveis aos saberes e dinâmicos na ativação de recursos ainda pouco conhecidos. Esses recursos, conforme demonstrado por várias experiências, podem estar pouco evidentes, e podem ser representados por "pessoas" ou por inumeráveis espaços da comunidade - formais ou informais - capazes de ativar a capacidade dos sujeitos de gerar sentido para suas vidas. Mediante a necessidade de processar avaliações e ações mais complexas, a valorização de estratégias não padronizadas ganharia sentido transformador. Consequentemente, a organização do trabalho dos coletivos envolvidos com as ações de reabilitação em diferentes unidades assistenciais deveria ser reproposta no sentido de expressar a disposição de produzir mudanças nas mais diferentes modalidades de assistência.

Em um contexto de inovação e complexificação das ações de reabilitação, noções desenvolvidas pela Reabilitação Psicossocial poderiam subsidiar ações coerentes. Por exemplo, poderiam ser tomadas algumas concepções que interagem na proposição de uma reabilitação cuja estratégia é voltada a desenvolver o aumento da capacidade contratual das pessoas portadoras de deficiência, ou seja, a capacidade de realizar negociações. Para Saraceno (1999), as negociações consistem em trocas materiais e afetivas, e estas precedem as relações. Precedem porque somente a existência de espaços de trocas sociais reais podem gerar relações, ao passo em que a existência de possibilidades para o desenvolvimento de relações não gera espaços reais de troca, ou seja, não cria as condições para que se realizem. Por sua vez, a possibilidade das redes de negociação aumentarem a participação e a contratualidade não ocorre em razão das pessoas entrarem fortalecidas - ou "habilitadas" - na negociação, mas porque as regras da negociação são alteradas no sentido de promover a participação de fortes e fracos em permanente dinâmica de trocas de interesses e competências.

Dessa forma concebida, a reabilitação consistiria em um conjunto de estratégias que acompanham a criação de espaços negociáveis, para o paciente, sua família, para a comunidade circundante e para os serviços que se ocupam do paciente. E assim, não caberia pensar a participação social como um produto a ser alcançado ao fim de um processo que supostamente habilita o sujeito para ela. A reabilitação ocorreria na participação e não para a participação. A reaquisição de uma habilidade motora, podendo estar presente no processo, não seria promovida como um fim, mas como um dos meios possíveis para realizar projetos de vida participante. Da mesma forma, tecnologias de natureza diversa seriam bemvindas, desde que empregadas enquanto instrumentos que atendem a decisões tomadas no contexto da insubstituível relação entre a pessoa com deficiência e os profissionais que a acompanham.

Por fim, seria necessário admitir que a reabilitação requer financiamento adequado. A natureza dos problemas condicionados pelas deficiências, sua cronicidade e a complexidade que envolve a vida nessa condição, implica em atenção prolongada, senão indefinidamente regulares, o que gera a necessidade de que os serviços cumpram uma agenda, por um lado muito abrangente em termos de vigilância a agravos e intercorrências de diferentes tipos ao tratar de coletivos e, por outro, extremamente individualizada, ao atender a singularidades tão complexas.

\footnotetext{
"Esse tema foi tratado mais extensamente no cap. 4 "Repensar a reabilitação - elementos para discussão", na tese "Saúde e reabilitação de pessoas com deficiência: políticas e modelos assistenciais".
} 
ALMEIDA, M. C. de.; CAMPOS, G.W.S. Policies and assistencial models in disabled's health and rehabilitation in Brazil: analisys about two decades. Rev. Ter. Ocup. Univ. São Paulo, v. 13, n. 3, p. 11826, set./dez. 2002.

\begin{abstract}
S: Despite the fact that in the last decades the physically impaired have achieved much regarding the brazilian legislation, the public policies have not offered the support and the possibilities for the consolidation of the exercise of their social rights. The study made focused on policies and assistance models in health and rehabilitation of the physically impaired, included in the brazilian assistance scenario in the last 20 years, with the objective of rebuilding and analysing their conceptual and practical paths. Based on data collected in books and periodicals, it focused on the hegemonical model, that being the one that concentrates the actions on the Rehabilitation Center, and the Rehabilitation based in the community - RBC, that was created as an alternative for the institutional practices, facing analytical references on the Communal Health. In the article, it was chosen to present results that could be articulated and used on the discussion around the development of the attention to the health and rehabilitation in the Sistema Único de Saúde - SUS. This way, the presentation of the analysis of the two models, that resulted in the consideration that any policies and assistance models that overcame the transference of public resources to the private sector, didn't take place in the period mentioned, in addition to the limitation of the biomedical paradigm, that leads to the the discussion of the proposals derived from the processes presented, considered of being potentially able of generating transformations in the attention to the health and rehabilitation of the physically impaired in the public sphere. The need for the viabilization of the expansion of the object of the rehabilitation action has been stressed, invoking notions of the psychosocial rehabilitation that estimulate the flexibilization and diversification of the services, to the realization of actions directed to the prevention or to the elimination of social exclusion processes, and also to the activation of non-established resources.
\end{abstract}

KEYWORDS: Disabled persons/rehabilitation. Policy making. Rehabilitation/trends. SUS (BR). Social support. Delivery of health care.

\title{
REFERÊNCIAS
}

\begin{abstract}
ALMEIDA, M.C. A pessoa portadora de deficiência física em seu cotidiano: reflexos e reflexões sobre a reabilitação. 1993. 117f. Dissertação (Mestrado). Instituto de Psicologia, Universidade de São Paulo, São Paulo, 1993.
\end{abstract}

BARDIN, L. Análise de conteúdo. Lisboa: Edições 70, 1979.

BRASIL. Ministério da Saúde. Secretaria de Assistência à saúde. Coordenação de Atenção a Grupos Especiais. Programa de Atenção à Saúde da Pessoa Portadora de Deficiência. Atenção à pessoa portadora de deficiência no Sistema Único de Saúde: planejamento e organização de serviços. Brasília, 1995.

CAMPOS, G. W. S. A reforma da reforma: repensando a saúde. São Paulo: HUCITEC, 1992.

CRISHNA, B. What is community- based rehabilitation? A view from experience. Child Care Health Dev., v. 25, n. 1, p. 27-35, 1999.

DE LA BELLACASA, R.P. Concepciones, paradigmas y evolución de las mentalidades sobre la discapascidad. In: REAL Patronato de prevención y de atención a personas com minusvalia. Discapacidad y información. Madrid, 1990. p. 27-61. Documento 11.
HELANDER, E. ; MENDIS, P.; NELSON, G. Training disabled people in the community. Geneve: World Health Organization, 1980.

MOMM, W.; KÖNIG, A. De la rehabilitación basada en la comunidad a los programas de integración comunitaria. Nuevo concepto de servicios para personas incapacitadas: experiencias y reflexiones. Ginebra: Oficina Internacional del Trabajo, 1990.

NALLIN, A. A reabilitação em instituição: suas razões e procedimentos. Análise de representação no discurso. 1992. 282f. Dissertação (Mestrado). Instituto de Psicologia, Universidade de São Paulo, São Paulo, 1992.

OLIVER, F. C. Saúde mental e saúde da pessoa com deficiência: estudo do processo de incorporação de assistência pelos serviços municipais de saúde numa região do município de São Paulo (1989-1995). 1998. 194f. Tese (Doutorado). Faculdade de Saúde Pública, Universidade de São Paulo, São Paulo, 1998.

ORGANIZAÇÃO DAS NAÇÕES UNIDAS Programa de ação mundial para as pessoas com deficiência. São Paulo: CEDIPOD, 1992. 
ORGANIZACIÓN MUNDIAL DE LA SALUD. Comité de expertos de la OMS en prevención de incapacidades y rehabilitación. Ginebra, 1981. (OMS. Serie de Informes Técnicos, 668).

ORGANIZACIÓN MUNDIAL DE LA SALUD (OMS). Rehabilitación basada en la comunidad. Washington, 1989. (Guías tecnicas, 1).

ORGANIZACIÓN PANAMERICANA DE LA SALUD (OPS). Desarrollo y fortalecimiento de los sistemas locales de salud en la transformación de los sistemas nacionales de salud. los servicios de rehabilitación. Washington, 1993.

ORGANIZACIÓN PANAMERICANA DE LA SALUD (OPS). La rehabilitación por discapacidad en América Latina y el Caribe. Bol. Oficina Sanit. Panamer., v. 120, n. 4, p. 358-61, 1996.

Recebido para publicação: 23/05/2002

Aceito para publicação: 10/07/2002
PEAT, M. Community based rehabilitation . London: W. B. Saunders, 1997.

ROCHA, E. F. Corpo deficiente: em busca da reabilitação? Uma reflexão a partir da ótica das pessoas portadoras de deficiência física. 1990.310p. Dissertação (Mestrado). Instituto de Psicologia, Universidade de São Paulo, São Paulo, 1990.

SARACENO, B. Libertando identidades. Da reabilitação psicossocial à cidadania possível. Belo Horizonte: Instituto Franco Basaglia/Te Corá, 1999.

SOARES, L. T. B. Terapia ocupacional: lógica do capital ou trabalho? Retrospectiva da profissão no estado brasileiro de 1950 à 1980. 1987. 234p. Dissertação (Mestrado). Centro de Educação e Ciências Humanas, Universidade Federal de São Carlos, São Carlos, SP, 1987. 